\title{
Cirurgia de alta frequência no colo uterino: análise dos fatores de recidiva
}

\section{Loop electrosurgical excision procedure: analysis of recurrence factors}

Pâmela Mendes Arruda․ Raquel Autran Coelho². Karinne Cisne Fernandes Rebouças ${ }^{3}$. Amanda Zélia de Sousa Tavares ${ }^{4}$. Gabriele Santana Sá Lima ${ }^{4}$.

1 Residente do Programa de Ginecologia e Obstetrícia pela Universidade Federal do Ceará (UFC), Médica da Maternidade Escola Assis Chateaubraind (MEAC), Fortaleza, Ceará, Brasil. 2 Orientadora, Supervisora do Programa de Ginecologia e Obstetrícia, Maternidade Escola Assis Chateaubraind (MEAC), Fortaleza, Ceará, Brasil. 3 Ginecologista e Obstetra, Maternidade Escola Assis Chateaubraind (MEAC), Fortaleza, Ceará, Brasil. 4 Aluna do curso de graduação de Medicina, Universidade Federal do Ceará (UFC), Fortaleza, Ceará, Brasil.

\section{RESUMO}

Introdução: o tratamento da lesão intraepitelial de alto grau do colo uterino (LIEAG), precursora de carcinoma invasor, é realizado preferencialmente por conização. Objetivo: correlacionar a evolução clínica de pacientes acometidas por LIEAG submetidas a conização com dados do espécime cirúrgico e com fatores epidemiológicos. Metodologia: estudo retrospectivo de 60 pacientes submetidas a conização por cirurgia de alta frequência (CAF) de 2012 a 2015, cujo acompanhamento da evolução foi realizado com exame citopatológico e colposcópico. Foram excluídas as pacientes gestantes, imunossuprimidas, que tinham se submetido apenas à biópsia, conização a frio ou histerectomia e resultados anatomopatológicos sem descrição das margens cirúrgicas. Resultados: a medida da altura da peça variou de 0,5 a $3,3 \mathrm{~cm}$. Dez pacientes apresentaram recidiva. Não houve influência de idade ou fumo e contracepção. Houve diferença significativa entre a profundidade da peça e a ocorrência de margens comprometidas. Visando diminuir as complicações da conização, observa-se uma tendência a redução do tamanho das peças. Este estudo mostrou aumento do risco de margens comprometidas em tais situações, sem repercussão na recidiva de doença. Conclusão: a ocorrência de margens comprometidas foi maior em espécimes menores de conização, mas esse tamanho não influenciou no risco de recidiva de lesão induzida pelo HPV (Papiloma Vírus Humano).

Palavras-chave: Conização. Neoplasia intraepitelial. Recidiva.

\section{ABSTRACT}

Introduction: The treatment of high-grade squamous intraepithelial lesion (HSIL), precursor of invasive carcinoma, is performed preferably by conization. Objective: To correlate the clinical evolution of HSIL patients submitted to conization with data from the surgical specimen and with epidemiological factors. Methodology: Retrospective study of 60 patients submitted to Loop Electrosurgical Excision Procedure (LEEP) from 2012 to 2015, whose evolution follow-up was performed with cytopathological and colposcopic exams. Pregnant, immunosuppressed, patients who had undergone biopsy, cold conization or hysterectomy, and anatomopathological results without surgical margins were excluded. Results: The height of the piece varied from 0.5 to $3.3 \mathrm{~cm}$. Ten patients presented recurrence. There was no influence of age or smoking and contraception. There was a significant difference between the depth of the piece and the occurrence of compromised margins. In order to reduce the complications of the conization, there is a tendency to reduce the size of the pieces. This study showed an increased risk of margins compromised in such situations, however, without repercussion on disease recurrence. Conclusion: The occurrence of compromised margins was higher in smaller conization specimens, but this size did not influence the risk of recurrence of induced HPV lesion.

Keywords: Conization. Intraepithelial neoplasia. Recurrent.

Autor correspondente: Pâmela Mendes Arruda, Rua Itália, 1014, Maraponga, Fortaleza, Ceará. CEP: 60710-760. Telefone: +55 85 34956700/98738-5999. E-mail: pam_mendesarruda@yahoo.com.br

Conflito de interesses: Não há qualquer conflito de interesses por parte de qualquer um dos autores.

Recebido em: 09 Mar 2017; Revisado em: 21 Mai 2017; Aceito em: 25 Mai 2017. 


\section{INTRODUÇÃO}

O câncer de colo de útero é o terceiro mais frequente na população feminina e a quarta causa de morte de mulheres por câncer no Brasil.

Redução de $80 \%$ da mortalidade pôde ser alcançada pelo rastreamento de mulheres na faixa etária de risco e pelo tratamento da lesão precursora, a lesão intraepitelial cervical de alto grau (LIEAG). ${ }^{2} \mathrm{O}$ tratamento padrão para a LIEAG é a conização do colo uterino, sendo a conização cirúrgica com bisturi e a cirurgia de alta frequência (CAF), as técnicas mais utilizadas., ${ }^{2,3}$

A conização por CAF tem a vantagem de poder ser realizada em ambulatório, sob anestesia local, com menos sangramento e menor tempo cirúrgico. ${ }^{3} \mathrm{O}$ tamanho da peça removida varia conforme o julgamento médico, baseado nas características, no tamanho da lesão e no tipo de zona de transformação do colo do útero da paciente. ${ }^{4}$

A excisão completa da lesão é determinante no sucesso do tratamento. Fatores relacionados à neoplasia residual/ recorrência após conização seriam o tamanho da peça excisada, as margens cirúrgicas comprometidas, a idade da paciente, o tabagismo e o uso de métodos contraceptivos hormonais. ${ }^{2,5}$ A detecção desses possíveis fatores de risco poderia auxiliar na seleção para um seguimento mais intensivo ou até mesmo para a conduta intervencionista. ${ }^{6}$

O objetivo desse estudo é correlacionar a evolução clínica de pacientes submetidas a CAF, com dados do espécime cirúrgico e com fatores epidemiológicos.

\section{MATERIAIS E MÉTODOS}

Foi realizado estudo retrospectivo de 60 pacientes submetidas à conização por CAF na Maternidade Escola Assis Chateaubriand, de 2012 a 2015.

Para obtenção dos registros hospitalares das pacientes a serem incluídas no estudo, foram levantados no setor da patologia os resultados histopatológicos que incluíam produto de colo uterino. Encontraram-se 215 registros de produtos de biópsia do colo uterino.

Foram solicitados ao serviço de arquivo médico os prontuários referentes aos registros obtidos. Foram excluídas as pacientes gestantes, imunossuprimidas, que tinham se submetido apenas à biópsia, conização a frio ou histerectomia e resultados anatomopatológicos que não descreveram as margens cirúrgicas, totalizando 60 pacientes.

Os prontuários das pacientes foram revisados para obter os seguintes dados:idade; tabagismo; uso demétodos contraceptivos hormonais; resultado do citopatológico inicial de colo uterino (normal, ASC-US - atipias de significado indeterminado em células escamosas, possivelmente não neoplasicas, ASC-H atipais de significado indeterminado em células escamosas, não se pode excluir lesão intraepitelial de alto grau, LIEBG - lesão intraepitelial de baixo grau, LIEAG - lesão intraepitelial de alto grau, AGC- células glandulares atípicas, adenocarcinoma in situ e carcinomas invasores); resultado histopatológico do CAF (cervicite crônica, metaplasia escamosa, neoplasia intraepitelial grau 1 (NIC 1), neoplasia intraepitelial grau 2/3 (NIC 2/3), carcinoma escamoso invasivo, adenocarcinoma in situ e adenocarcinoma invasor); altura da peça do CAF e avaliação de suas margens, se livres ou comprometidas pela doença. Foi avaliado o seguimento dessas pacientes no primeiro ano pós-conização, com citologia e colposcopia semestrais. Foi considerado recidiva de doença a presença de citologia evidenciando LIEAG e ASC-H no seguimento.

O programa estatístico utilizado foi Sigmaplot. Para as variáveis de natureza quantitativa, foram calculadas medidas-resumo, como média. Shapiro-Wilk test foi utilizado para avaliar a correlação entre: idade, uso de métodos contraceptivos hormonais e tabagismo com a recidiva de doença; altura da peça do CAF com as margens cirúrgicas do CAF e com a recidiva de doença; status cirúrgico da margem do CAF com a recidiva de doença.

Esse projeto foi aprovado pelo Comitê de Ética em Pesquisa da Maternidade Escola Assis Chateaubriand da Universidade Federal do Ceará, dentro das normas que regulamentam a pesquisa em seres humanos, do Conselho Nacional da Saúde - Ministério da Saúde, Resolução no 196 de 10 de outubro de 1996 (DOU de 16 de outubro de 1996).

\section{RESULTADOS}

A média da idade das pacientes foi de 35,4 anos. 49 pacientes $(81,6 \%)$ apresentavam laudo citológico inicial de LIEAG, 4 $(6,7 \%)$ LIEBG, 5 (8,3\%) ASC-US, 1 (1,7\%) ACG e 1 (1,7\%) normal. Nas pacientes sem citologia com LIEAG, CAF foi indicado por discordância cito-histológica ou por lesão de baixo grau persistente adentrando o canal.

Em relação ao resultado histopatológico do CAF, observou-se $39(65 \%)$ NIC II/III, 14 (23,3\%) NIC I, 4 (6,7\%) cervicites crônicas e $3(5 \%)$ Carcinomas de células escamosas.

A altura da peça do CAF variou de 0,5 a $3,3 \mathrm{~cm}$. Trinta e três (55\%) peças de CAF apresentaram margens comprometidas por doença.

Oito pacientes não retornaram para o seguimento e as 3 pacientes com carcinoma foram encaminhadas para o serviço de oncologia. Das 49 pacientes que permaneceram no seguimento, 35 realizaram duas citologias de controle e 14 realizaram apenas a primeira citologia de controle, totalizando uma perda de $22(36 \%)$ pacientes no seguimento.

Dez pacientes $(20,4 \%)$ apresentaram recidiva da doença. Não houve influência de idade, do uso de contracepção hormonal e do tabagismo na recidiva de doença (Tabela 1).

Houve diferença significativa entre a altura da peça e a ocorrência de margens comprometidas. Não houve diferença significativa entre a altura do CAF e recidiva, nem entre o status cirúrgico das margens do CAF e recidiva (Tabela 2). O ponto de corte para o achado de margens comprometidas foi em torno de $12 \mathrm{~mm}$ (Gráfico 1). 
Tabela 1. Correlação da idade, ACH e tabagismo na recidiva (citologia de controle).

\begin{tabular}{lcccc}
\hline \multicolumn{5}{c}{ Citologia de Controle } \\
\hline Idade (anos)* & Negativa & LIEBG & LIEAG/ASC-H & Valor $p$ \\
ACH** & $33,4 \pm 9,8$ & $31,2 \pm 8,4$ & $40,4 \pm 12,2$ & 0,122 \\
Sim & $12(37,5 \%)$ & $4(57,2 \%)$ & $6(60 \%)$ & \\
Não & $20(62,5 \%)$ & $3(42,8 \%)$ & $4(40 \%)$ & 0,358 \\
Tabagismo** & & & \\
Sim & $7(21,9 \%)$ & $1(14,3 \%)$ & $1(10 \%)$ & 0,668 \\
Não & $25(78,1 \%)$ & $6(85,7 \%)$ & $9(90 \%)$ & \\
\hline
\end{tabular}

*Média \pm D.P.; ** N (\%)

Tabela 2. Associação da altura do CAF e do status cirúrgico das margens do CAF na recidiva (citologia de controle).

\begin{tabular}{lcccc}
\hline \multicolumn{5}{c}{ Citologia de Controle } \\
\hline Altura do CAF $(\mathrm{cm})^{*}$ & Negativa & LIEBG & LIEAG/ASC-H & Valor $\mathrm{p}$ \\
Margens** & $1,47 \pm 0,6$ & $1,37 \pm 0,7$ & $1,40 \pm 0,6$ & 0,898 \\
Livres & $12(37,5 \%)$ & $2(28,5 \%)$ & $6(60 \%)$ & 0,349 \\
Comprometidas & $20(62,5 \%)$ & $5(71,5 \%)$ & $4(40 \%)$ & \\
\hline
\end{tabular}

*Média \pm D.P.; ** N (\%)

Gráfico 1. Valor de corte para ocorrência de margens livres em amostras de CAF.

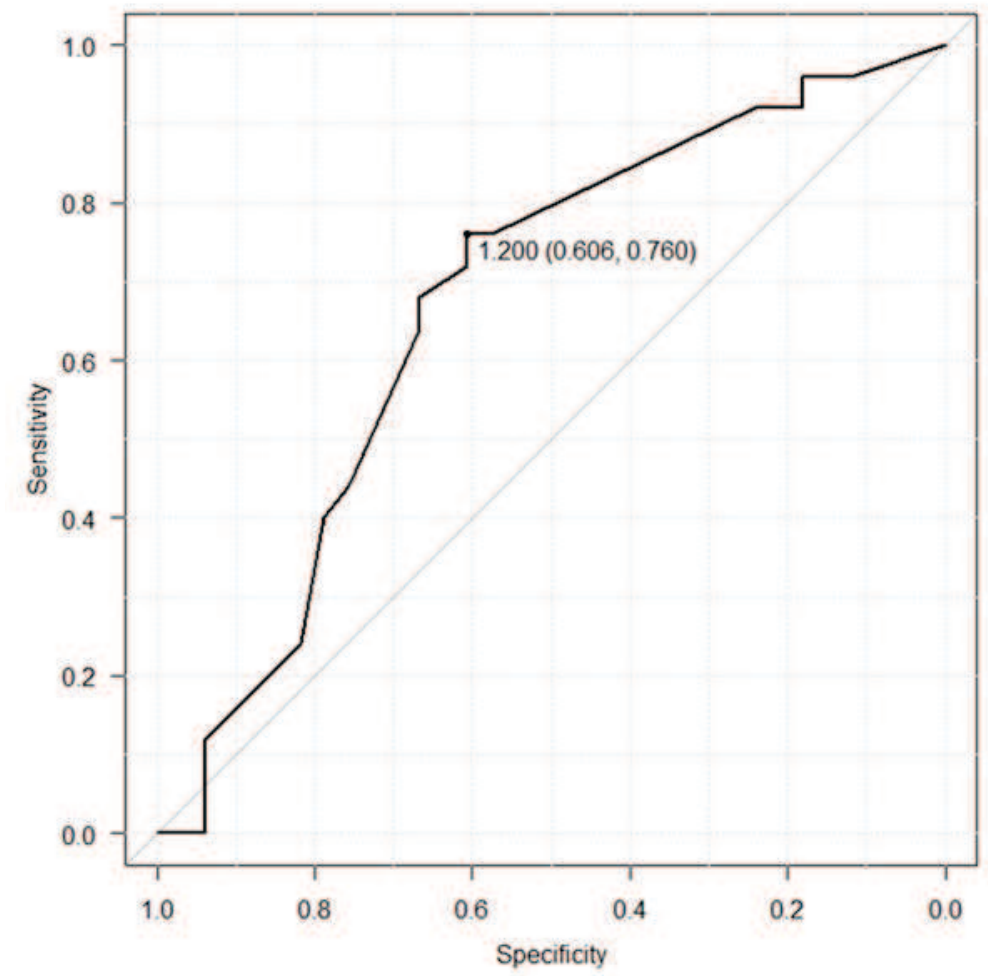




\section{DISCUSSÃO}

O tabagismo, o uso de contraceptivos hormonais e a idade da paciente têm sido elencados como fatores relacionados à recorrência de doença em pacientes com LIEAG submetidas a conização, assim como a altura da peça excisada e o comprometimento cirúrgico de suas margens.

Apesar do adequado tratamento da lesão precursora, a recorrência ocorrerá, em média, em 5 a 64\% dos casos, podendo variar conforme o tratamento realizado e a população estudada. ${ }^{6}$ Neste estudo, a taxa de recidiva encontrada foi de $20,4 \%$.

Estudos observaram que as pacientes fumantes apresentavam risco maior de falha no tratamento. $\mathrm{O}$ tabagismo parece influenciar na resposta ao tratamento, devendo essas pacientes ser submetidas a seguimento mais rigoroso. ${ }^{7,8}$ Em relação ao uso de métodos contraceptivos hormonais e recidiva da doença, estudo mostrou que o uso da medicação não aumentou a taxa de recorrência de lesões após o tratamento destrutivo ou cirúrgico. ${ }^{9}$ Há evidências de que a idade influencia no índice de recorrência da lesão. ${ }^{10,11}$ Ainda não está claro por que as mulheres mais velhas podem ter mais probabilidade de experimentar persistência/recorrência. Uma possível razão pode ser a imunidade alterada ou seleção temporal para vírus com maior risco oncogênico. ${ }^{12}$ Em relação a esses fatores, nosso estudo não evidenciou diferença significativa na evolução das pacientes tratadas por NIC.

No que diz respeito ao tratamento das lesões precursoras do câncer de colo uterino, a excisão completa da lesão parece ser determinante no sucesso da terapêutica. Entretanto, não há, na área da patologia cervical, uma definição da quantidade mínima de canal cervical a ser retirado na conização, possibilitando uma chance de ressecção incompleta. ${ }^{13,14}$ Contudo, é sabido que quanto maior a altura do cone, maior o risco de estenose, sangramento, parto prematuro, baixo peso e morte perinatal nas gestações subsequentes. ${ }^{15}$ Visando diminuir tais complicações, observa-se uma tendência à redução do tamanho das peças, entretanto nossa análise evidenciou que há um aumento do risco das margens comprometidas em tais situações.

\section{REFERÊNCIAS}

1. Instituto Nacional de Câncer José Alencar Gomes da Silva (INCA). Colo do útero [Internet]. Rio de Janeiro: INCA; 2016 [Acesso em: 06 fev 2016]. Disponível em: http://www2.inca.gov.br/wps/wcm/ connect/tiposdecancer/site/home/colo_utero

2. Fonseca FV, Tomasich FD, Jung JE. High-grade intraepithelial cervical lesions: evaluation of the factors determining an unfavorable outcome after conization. Rev Bras Ginecol Obstet. 2011;33(11):33440 .

3. Juliato CR, Teixeira JC, Derchain SF, Barbosa SB, Martinez EZ, Panetta K, et al. Correlation between the histological report of biopsy and conization by the Loop Electrosurgical Excision Procedure (LEEP) in the treatment of cervical intraepithelial neoplasia. Rev Bras Ginecol Obstet. 2000;22(2):65-70.
Milinovic et al (2009) não encontraram margens endocervicais comprometidas em nenhum cone com mais de $18 \mathrm{~mm}$ de profundidade, sugerindo ser esta a altura máxima a ser ressecada para minimizar as futuras complicações obstétricas. ${ }^{16}$ Em nossa análise, o ponto de corte para o achado de margens comprometidas foi em torno de $12 \mathrm{~mm}$. Contudo, a importância do envolvimento das margens no estabelecimento do risco de doença pós-tratamento ainda é controversa. ${ }^{17}$ Diversos estudos têm mostrado a presença de margens positivas como um fator preditor de doença persistente, mas as frequências citadas na literatura são variáveis, com valores entre 7,2\% a 42,5\%. ${ }^{18,19}$ Por outro lado, em 2004, Chávez e Castro não encontraram relação entre as margens cirúrgicas e o risco de recidiva, sugerindo o mesmo seguimento para pacientes independente do status das margens cirúrgicas. ${ }^{20}$

$\mathrm{Na}$ literatura, a frequência de lesões persistentes em pacientes com margem comprometida varia de $22 \%$ para $78 \%{ }^{21}{ }^{21}$ Em nossa série, apresentamos $13,8 \%$ de recorrências para pacientes com margens afetadas, $86,2 \%$ dos casos com margens positivas não desenvolveram doença persistente/recorrente. Não houve, em nosso estudo, relação estatisticamente significativa entre margens afetadas e doença persistente, apoiando estudos anteriores que indicam que o estado de margens de ressecção tem utilidade limitada na predição de recidiva.

Nosso estudo apresentou elevada taxa de perda de seguimento das pacientes após o procedimento. Tal fato também é descrito na literatura, com relato de perda no seguimento de $43 \%$ das pacientes em estudo semelhante. ${ }^{22}$ Esse dado leva a reflexão sobre necessidade de individualização quanto a decisão de seguimento de mulheres com margens comprometidas, apesar de ainda existirem divergências na literatura.

\section{CONCLUSÃO}

A ocorrência de margens comprometidas foi maior em espécimes menores de conização por CAF, mas esse tamanho não influenciou no risco de recidiva de lesão HPV induzida. A recidiva de lesão HPV induzida após conização por CAF não foi influenciada pela idade das mulheres, tabagismo ou uso de contracepção hormonal. Houve alta perda de seguimento da evolução após o procedimento.

4. Sarian LO, Derchain SF, Pitta DR, Morais SS, Rabelo-Santos SH. Factors associated with HPV persistence after treatment for highgrade cervical intraepithelial neoplasia with large loop excision of the transformation zone (LLETZ). J Clin Virol. 2004;31(4):270-4.

5. Saeaib N, Boonyapipat S, Tungsinmunkong K, Liabsuetrakul T. Predictors of the residual disease of high-grade lesions and microinvasive squamous cell carcinoma of the cervix following conization. J Med Assoc Thai. 2009;92(11):1406-12.

6. Ghaem-Maghami S, Sagi S, Majeed G, Soutter WP. Incomplete excision of cervical intraepithelial neoplasia and risk of treatment failure: a meta-analysis. Lancet Oncol. 2007;8(11):985-93.

7. Acladious NN, Sutton C, Mandal D, Hopkins R, Zaklama M, 
Kitchener H. Persistent human papillomavirus infection and smoking increase risk of failure of treatment of cervical intraepithelial neoplasia (CIN). Int J Cancer. 2002;98(3):435-9.

8. Giuliano AR, Sedjo RL, Roe DJ, Harri R, Baldwi S, Papenfuss $\mathrm{MR}$, et al. Clearance of oncogenic human papillomavirus (HPV) infection: effect of smoking (United States). Cancer Causes Control 2002;13(9):839-46.

9. Frega A, Scardamaglia P, Piazze J, Cerekja A, Pacchiarotti A, Verrico M, et al. Oral contraceptives and clinical recurrence of human papillomavirus lesions and cervical intraepithelial neoplasia following treatment. Int J Gynecol Obstet. 2008;100(2):175-8.

10. Verguts J, Bronselaer B, Donders G, Arbyn M, Van Eldere J, Drijkoningen M, Poppe W. Prediction of recurrence after treatment for high-grade cervical intraepithelial neoplasia: the role of human papillomavirus testing and age at conisation. BJOG. 2006;113(11):1303-7.

11. Lu CH; Liu FS; Kuo CJ; Chang CC; Ho ES. Prediction of persistence or recurrence after conization for cervical intraepithelial neoplasia III. Obstet Gynecol. 2006;107(4):830-5.

12. Zhu M, He Y, Baak JP, Zhou X, Qu Y, Sui L, et al. Factors that influence persistence or recurrence of high-grade squamous intraepithelial lesion with positive margins after the loop electrosurgical excision procedure: a retrospective study. BMC Cancer. 2015;15(1):744-54.

13. Duggan BD, Felix JC, Muderspach LI, Gebhardt JA, Groshen $\mathrm{S}$, Morrow $\mathrm{CP}$, et al. Cold-knife conization versus conization by the loop electrosurgical excision procedure: a randomized, prospective study. Am J Obstet Gynecol. 1999;180(2):276-82.

14. Freitas F, Menke CH, Rivoire WA, Passos EP, cols. Rotinas em Ginecologia. 6. ed. Porto Alegre: Artmed; 2011. Capítulo 27, Analgesia e anestesia em obstetrícia; p. 395-400.
15. Baldauf JJ, Dreyfus M, Ritter J, Meyer P, Philippe E. Risk of cervical stenosis after large loop excision or laser conization. Obstet Gynecol. 1996;88(6):933-8.

16. Milinovic D, Kalafatic D, Babic D, Oreskovic LB, Grsic HL, Oreskovic S. Minimally invasive therapy of cervical intraepithelial neoplasia for fertility preservation. Pathol Oncol Res. 2009;15(3):5215.

17. Lubrano A, Medina N, Benito V, Arencibia O, Falcón JM, Leon L, et al. Follow-up after LLETZ: a study of 682 cases of CIN 2CIN 3 in a single institution. Eur J Obstet Gynecol Reprod Biol. 2012;161(1):71-4.

18. Nuovo J, Melnikow J, Willan AR, Chan BK. Treatment outcomes for squamous intraepithelial lesions. Int $\mathrm{J}$ Gynaecol Obstet. 2000;68:25-33.

19. Maluf PJ, Adad SJ, Murta EF. Outcome after conization for cervical intraepithelial neoplasia grade III: relation with surgical margins, extension to the crypts and mitoses. Tumori. 2004;90(5):473-7.

20. Zamudio MA, Castro LS. Comparison of the evolution of women with cervical intraepithelial neoplasia degree III treated with cervical cone: negative vs positive surgical margin. Ginecol Obstet Mex. 2004;72(6):279-82.

21. White CD, Cooper WL, Williams RR. Cervical intraepithelial neoplasia extending to the margins of resection in conization of the cervix. J Reprod Med. 1991;36(9):635-8.

22. Araújo CF. Neoplasia intraepitelial cervical: envolvimento das margens de ressecção, após excisão eletrocirúrgica com alça, e recidiva da doença [Internet]. Salvador: Universidade Federal da Bahia; 2013 [acesso em: 05 jan 2017]. Disponível em: http:// repositorio.ufba.br/ri/handle/ri/14248

\section{Como citar:}

Arruda PM, Coelho RA, Rebouças KC, Tavares AZ, Lima GS. Cirurgia de alta frequência no colo uterino: análise dos fatores de recidiva. Rev Med UFC. 2017 set-dez;57(3):30-34. 